\title{
Aktuelles aus Ausbildung, Forschung und Begutachtung
}

\section{Paul Drobec}

Online publiziert: 25. Juni 2012

(c) Springer-Verlag Wien 2012

Sehr verehrte Kolleginnen und Kollegen! Werte Leser!

Im vorliegenden Heft finden Sie vier interessante Originalarbeiten über sehr unterschiedliche Gebiete der Augenheilkunde:

Die Augenklinik Graz stellt ihr freigegebenes Curriculum betreffend die Facharztausbildung im Teilbereich der Strabologie vor. Es besteht aus einem klar strukturierten Ablauf der 6-monatigen Ausbildung. Der Lernzielkatalog entspricht internationalem Niveau, und die Ausbildungskriterien halten sich gemäß dem Qualitätssicherungsmodell der European Foundation for Quality Management (EFQM) an die internationalen Richtlinien der International Commission for Optics (ICO) und des European Board of Ophthalmology (EBO).

Der Beitrag der Augenklinik Szeged befasst sich mit dem Keratokonus und seiner Verlaufsbeobachtung mittels Ultraschallbiomikroskopie und weist die guten Erfolge sowohl beim Tragen von formstabilen Kontaktlinsen als auch bei perforierender Keratoplastik nach.

Die Arbeit aus der Augenklinik Innsbruck handelt von der Erstellung augenfachärztlicher Gutachten und erörtert die Grundlagen für den Grad der Invalidität bzw. Minderung der Erwerbsfähigkeit.

Der Beitrag aus der Augenabteilung Linz erörtert die Ergebnisse nach Descemet-Membran-Endothelkeratoplastik, welche zu einer guten visuellen Rehabilitation führt.
Schließlich darf ich auf die Berichte über unsere 53. Tagung verweisen, welche vom 16.-19. Mai 2012 in Villach stattgefunden hat. Fast 500 Besucher erlebten ein hochstehendes wissenschaftliches Programm, besuchten eine groß angelegte Industrieausstellung und hatten reichlich Gelegenheit, kollegiale Kontakte zu vertiefen. Zahlreiche Preise an junge Kollegen konnten vergeben werden. In meiner Eröffnungsrede bin ich auf standespolitische Aspekte eingegangen. Hernach durfte ich die Laudatio für Frau Prim. Prof. Dr. Susanne Binder halten, die von der Österreichischen Ophthalmologischen Gesellschaft (ÖOG) in einer Urabstimmung zu ihrem Ehrenmitglied gewählt wurde. Wir dürfen unserer ChefHerausgeberin dazu herzlichst gratulieren!

Ich darf Ihnen viel Freude mit diesen interessanten Beiträgen des vorliegenden Heftes wünschen und bin

mit kollegialen Grüßen,

MR. Prim. Prof. Dr. Paul Drobec

Associate Editor

\section{Interessenskonflikt}

Es besteht kein Interessenskonflikt. 